\title{
VALIDEZ DE CONSTRUCTO DE UN TEST PARA MEDIR EL NIVEL DE CUIDADO INFANTIL EN EDUCACIÓN PRIMARIA
}

\section{CONSTRUCT VALIDITY OF A TEST TO MEASURE THE LEVEL OF CHILD CARE IN PRIMARY EDUCATION}

\author{
Oscar Luis Ochoa Martínez \\ Universidad Pedagógica de Durango
}

\section{Ruth Villanueva García}

Instituto Universitario Anglo Español

México

\section{RESUMEN}

Este trabajo de investigación tuvo como objetivo construir y determinar la validez de constructo de un Test para medir el nivel de "cuidado infantil" en niños de educación primaria; el proceso de construcción del Test inició con la propuesta de 21 ítems cuyo contenido contemplo dimensiones de la Psicología Positiva, entre ellas, el apoyo económico, el cuidado de la salud, el bienestar emocional y la sana convivencia; estos ítems

\footnotetext{
1 Doctor en Gestión Humanista desde una Perspectiva Humanista, Profesor en la Universidad Pedagógica de Durango, en Durango Dgo., México. Contacto: chokar128@ hotmail.com ORCID: https://orcid.org/0000-0003-3330-9138 2 Maestra en Ciencias de la Educación, Profesora de Educación Primaria en Durango, Dgo, México. Contacto: rthvillanueva@yahoo.com ORCID: https://orcid.org/00000001-6906-9733
}

fueron expuestos a la valoración de expertos, obteniendo como resultado la eliminación de seis ítems; los 15 restantes fueron objeto de medida de sus propiedades psicométricas de fiabilidad y validez de constructo; la primera, mediante el estadístico del alfa de Cronbach, y la segunda, a través de la prueba de análisis factorial exploratorio (AFE). La muestra del estudio fue de tipo determinístico seleccionando a 154 niños que cursaban sus estudios de educación primaria en la zona escolar No. 13 en la Ciudad de Durango, Durango, México, en el ciclo escolar 2019-2020. El resultado de la aplicación del Test arrojó un valor del estadístico de 0.79 , mientras que la prueba de AFE confirmó la validez de constructo del Test y la existencia de las variables latentes: Integración familiar, 
Convivencia familiar, Atención a la salud, Tiempo de esparcimiento y Apoyo económico. Respecto al resultado global de "cuidado infantil" en esta muestra y contexto, se encontró un alto porcentaje de apoyo por parte de los familiares.

PALABRAS CLAVE. Validez de constructo, test y cuidado infantil

\section{ABSTRACT}

The objective of this research was to construct and determine the construct validity of a test to measure the level of "child care" in elementary school children; the construction process of the Test started with the proposal of 21 items whose content contemplated dimensions of Positive Psychology, among them, economic support, health care, emotional well-being and healthy coexistence; these items were exposed to expert assessment, obtaining as a result the elimination of six items; the remaining 15 were measured for their psychometric properties of reliability and construct validity; the first one, through Cronbach's alpha statistic, and the second one, through the exploratory factor analysis test (AFE). The study sample was deterministic, selecting 154 children who were studying in primary education in school zone 13 in Durango City, Durango, Mexico, in the 2019-2020 school year. The result of the application of the Test gave a statistical value of 0.79 , while the AFE test confirmed the validity of the Test construct and the existence of the latent variables: Family Integration, Family Coexistence, Health Care, Leisure Time and Economic Support. Regarding the overall result of "child care" in this sample and context, a high percentage of support from family members was found.

KEYWORDS. Construct Validity, test and child care

\section{INTRODUCCIÓN}

La educación primaria tiene como objetivo el desarrollo integral del niño y el núcleo familiar contiene las bases para lograrlo, en este sentido, el contexto familiar es vital tanto para la sociedad como para el desarrollo del ser humano, razón por la que la educación se concibe como tarea primordial de la familia, aunque compartida de una manera significativa en la escuela, su entorno y contexto social.

La familia es el primer transmisor de elementos culturales y primer agente de socialización, "Según Coleman, la familia no solo influye por su estatus económico, sino también por el apoyo fuerte y efectivo que puede brindar en la educación de los estudiantes (Coleman, 1966, citado en Razeto, 2016, p. 6).

En estas observaciones queda de manifiesto la importancia del cuidado que brinda la familia a los menores y del impacto que tiene en su rendimiento escolar, este fenómeno es atendido por la Psicología Positiva que trata de comprender la tarea educativa desde el punto de vista de las capacidades y fortalezas de las personas; al respecto de este campo de la psicología, (Seligman 1996, citado en Royo, 2012, p. 14) describe lo siguiente:

(...) destaca la necesidad de completar la investigación del comportamiento humano centrándose en los aspectos positivos y en los recursos internos y externos del individuo que facilitan su desarrollo y que tienen beneficios para las personas (tales como el bienestar, la satisfacción, el optimismo, la resiliencia, la inteligencia emocional, el humor, la sabiduría, la felicidad, las redes de apoyo, etc.).

Con base en estos rasgos de personalidad y aspectos de la forma de vida del niño, se planteó la necesidad de construir y validar un instrumento a través del cual se pudiera medir el nivel de "cuidado infantil" en relación al abandono físico o negligencia infantil del que puede ser objeto el alumno de educación primaria. 
El cuidado infantil está directamente relacionado con el desarrollo infantil integral y a decir de Caridad y López (2015), este concepto implica la sinergia de un conjunto de acciones que colocan en el centro al niño y a su familia, y que garantizan la atención de sus necesidades en el momento oportuno.

En el proceso de construcción del Test se consideraron categorías propias del "cuidado infantil", entre ellas las relacionadas con el apoyo económico, el cuidado de la salud, el bienestar emocional y la sana convivencia.

De esta forma, se conceptualiza al cuidado infantil como la acción de ayudar a un niño a desarrollarse y a facilitarle bienestar en su vida cotidiana. Engloba, por tanto, hacerse cargo del cuidado material que implica un trabajo, del cuidado económico que implica un costo económico y del cuidado psicológico que implica el desarrollo de un vínculo afectivo, emotivo, sentimental. (Batthyány, et al., 2014. p. 338)

Con este antecedente y en un primer momento, el Test quedó integrado por 21 ítems (ver apéndice 1), mismos que fueron sujetos a valoración por el método de jueceo de expertos; como resultado de este proceso se eliminaron los siguientes ítems: $R_{2}$ y $R_{4}$ porque se consideraron triviales, $R_{5}$ y $R_{10}$ por medir lo mismo que $R_{9}, R_{6}$ por medir lo mismo que $R_{8} y, R_{19}$ por medir lo mismo que $R_{21}$. Después de este ajuste, el Test quedó integrado por 15 ítems, mismos que fueron nuevamente reordenados (ver apéndice 2).

En este momento del trabajo de investigación y con el propósito de fortalecer la validez del Test, se tomó la decisión de someterlo a prueba del análisis factorial confirmatorio, detallando que los ítems del Test ofrecen respuesta de acuerdo a una escala Likert de cuatro niveles apropiados para medir el "cuidado infantil", estos se describen de la siguiente manera: nivel "1", apoyo al 100\%; nivel "2", apoyo al $80 \%$ nivel "3", apoyo al $60 \%$ nivel " 4 ", apoyo al $40 \%$ o menos.

\section{METODOLOGÍA}

De acuerdo al objetivo y características de este trabajo de investigación, el estudio se consideró de carácter instrumental dado que se consideran como pertenecientes a esta categoría todos los estudios encaminados al desarrollo de pruebas y aparatos, incluyendo tanto el diseño (o adaptación) como el estudio de las propiedades psicométricas de los mismos (Montero y León, 2007).

\section{MUESTRA DEL ESTUDIO}

La muestra del estudio que se utilizó para construir y validar el instrumento fue de 154 niños que realizaban sus estudios de quinto grado de educación primaria durante el ciclo escolar 2019-2020, de la zona escolar No. 13, de la Ciudad de Durango, Durango, México; cabe mencionar que la muestra fue seleccionada de acuerdo al criterio que especifica la participación de al menos cinco encuestados por ítem del instrumento y, para el caso en particular, el número de participantes en la aplicación del Test cumple a la perfección con la observación que encuentra fundamento en el trabajo realizado por Damiani y Vega (2019) quienes afirman que este número se considera un buen tamaño muestral para el análisis factorial.

\section{HERRAMIENTAS PARA EL ANÁLISIS DE LA INFORMACIÓN}

La clasificación y organización de los datos y las pruebas psicométricas del instrumento, se llevaron a cabo con el uso de la hoja de cálculo de Excel y el programa estadístico SPSS en su versión 22, respectivamente.

\section{PRUEBA DE FIABILIDAD}

Una vez que el Test fue aplicado a los integrantes de la muestra seleccionada, se procedió a determinar su propiedad psicométrica de fiabilidad $y$, considerando que la escala de medida fue de tipo Likert, se utilizó el coeficiente 
alfa de Cronbach, a este respecto Maese, et al., (2016) afirman que este parámetro estadístico mide la fiabilidad de consistencia interna, grado en que las respuestas son consistentes a través de los ítems dentro de una medición.

\section{PRUEBA DE VALIDEZ DE CONSTRUCTO.}

La medida psicométrica de validez de constructo del Test, se realizó mediante la prueba de Análisis Factorial Exploratorio (AFE), "El Análisis Factorial Exploratorio es una de las técnicas más usadas en el desarrollo, validación y adaptación de instrumentos de medida psicológicos" (Lloret, et al., 2014, p. 1151); esta técnica se desarrollo de acuerdo a las siguientes fases:

a) El primer resultado del análisis para sustentar el desarrollo del procedimiento, consideró el resultado del estadístico de Medida Kaiser-Meyer-Olkin (KMO) de adecuación de muestreo.

b) Un segundo resultado del análisis para sustentar el desarrollo del procedimiento, consideró el resultado de la prueba de esfericidad de Bartlett ya que a través de ella se contrasta la hipótesis nula de que la matriz de correlaciónes es igual, el planteamiento de hipótesis fue descrito de la siguiente manera:

$$
\begin{aligned}
& H_{0} \text { : no existen factores en el modelo } \\
& H_{i} \text { : si existen factores en el modelo }
\end{aligned}
$$

El resultado de la prueba se obtuvo mediante el contraste de hipótesis:

$$
\text { si "p"valor }<0.5 \text {, rechazar } H_{0}
$$

Luego de administrar el test a la muestra de investigación, y antes de emprender el análisis factorial debe determinarse si los ítems están suficientemente interrelacionados para que este método pueda aplicarse provechosamente (Comrey, 1973). Existen algunas pruebas estadísticas que pueden emplearse con esa finalidad, y las más utilizadas son el test de esfericidad de Bartlett y la medida de adecuación muestral de Kaiser-Mayer-Olikin (KMO). (Perez y Medrano, 2010, p. 61)

c) La proporción de varianza común a una variable descrita a través de la tabla de comunalidades.

Basándose en el modelo y en las hipótesis formuladas, es posible demostrar que la varianza de cada variable se puede descomponer en esa parte de la variabilidad que viene explicada por una serie de factores comunes con el resto de variables que se denominan comunalidad de la variable (Montoya, 2007)

d) El gráfico de sedimentación. Este gráfico nos ofrece una imagen visual de los posibles factores que pueden existir en el gráfico y la influencia de cada uno de ellos respecto a los demás; en relación a la aplicación de este análisis, Moliner (2017), argumenta que mediante el método Kaiser se determinan tantos factores como autovalores mayores que 1 existan.

e) Las cargas factoriales del modelo que se registraron en la matriz de componentes rotados, fue obtenida mediante el análisis de componentes principales y rotación Varimax; la aplicación de esta técnica genera el producto final del estudio pues a decir de Calderón, et al (2018), con ella es posible analizar la validez y fiabilidad de dicho cuestionario a través de un análisis factorial de componentes principales con rotación Varimax.

\section{RESULTADOS}

Prueba de fiabilidad

En la tabla número 1, se encuentra el resultado de la prueba de fiabilidad del Test integrado por los 15 ítems, ahí se aprecia el valor del estadístico del alfa de Cronbach es considerado como uno de los principales parámetros para la evaluación de consistencia interna de instrumentos de 
medición de tipo cuestionarios, para el caso se obtuvo un valor de 0.79 , valor "muy bueno" de acuerdo a Cascaes, et al., (2015), quienes afirman que la literatura apunta a un valor mínimo de 0,70 y los valores preferenciales son entre 0.80 y 0.90 .

Tabla 1. Resultado de fiabilidad del Test

\begin{tabular}{|c|c|}
\hline \multicolumn{2}{|c|}{ Estadísticas de fiabilidad } \\
\hline $\begin{array}{c}\text { Alfa de } \\
\text { Cronbach }\end{array}$ & $\begin{array}{c}\mathrm{N} \text { de } \\
\text { elementos }\end{array}$ \\
\hline .793 & 15 \\
\hline
\end{tabular}

Prueba de Kaiser-Meyer-Olkin (KMO).

Una de las pruebas determinantes para validar el proceso del análisis factorial exploratorio del Test, fue el resultado de la prueba de medida de Kaiser-Meyer-Olkin (KMO); en la tabla número 2 se observa que el valor del estadístico fue de 0.76 , valor que se considera como "aceptable" y que avala la ejecución para la continuidad del proceso de análisis.

De igual manera, en la tabla también se observa el resultado de la prueba de esfericidad de Bartlett que se realiza a través del estadístico Chi-cuadrado y, de acuerdo al contraste de hipótesis planteado, el resultado indicó una significancia estadística, motivo por el cual se rechazó la hipótesis nula de similitud de la matriz.

Tabla 2. Resultado de la Prueba de KMO y Bartlett

\begin{tabular}{|l|l|r|}
\hline \multicolumn{3}{|c|}{ Prueba de KMO y Bartlett } \\
\hline \multicolumn{2}{|l|}{$\begin{array}{l}\text { Medida Kaiser-Meyer-Olkin de } \\
\text { adecuación de muestreo }\end{array}$} & .768 \\
\hline $\begin{array}{l}\text { Prueba de } \\
\text { esfericidad } \\
\text { de Bartlett }\end{array}$ & $\begin{array}{l}\text { Aprox. Chi- } \\
\text { cuadrado }\end{array}$ & 801.999 \\
\cline { 2 - 3 } & gl & 210 \\
\cline { 2 - 3 } & Sig. & .000 \\
\hline
\end{tabular}

\section{PROPORCIÓN DE VARIANZA COMÚN}

En la tabla número 3, se muestra la proporción de varianza común dentro de una variable y como el análisis de componentes principales funciona bajo el supuesto inicial de que toda variación es común, entonces, antes de la extracción los valores de las comunalidades son todas de valor "1", una vez extraídos los factores se tiene una mejor idea en cuanta varianza es en realidad común, como ocurrió en el caso del análisis del Test donde se obtuvieron valores aceptables para la propuesta del modelo en cada uno de los ítems.

Tabla 3. Valores de las comunalidades

\begin{tabular}{|l|c|c|}
\hline \multicolumn{3}{|c|}{ Comunalidades } \\
\hline & Inicial & Extracción \\
\hline $\begin{array}{l}\text { R1 Aportan dinero } \\
\text { para mis festividades } \\
\text { escolares }\end{array}$ & 1.000 & .652 \\
\hline $\begin{array}{l}\text { R2 Me apoyan con } \\
\text { recursos para realizar } \\
\text { mis tareas }\end{array}$ & 1.000 & .460 \\
\hline $\begin{array}{l}\text { R3 Se preocupan por } \\
\text { mi higiene }\end{array}$ & 1.000 & .602 \\
\hline $\begin{array}{l}\text { R4 Se preocupan por } \\
\text { mi aspecto personal }\end{array}$ & 1.000 & .705 \\
\hline $\begin{array}{l}\text { R5 Se preocupan } \\
\text { porque coma } \\
\text { sanamente }\end{array}$ & 1.000 & .666 \\
\hline $\begin{array}{l}\text { R6 Se preocupan por el } \\
\text { tiempo que paso en TV } \\
\text { y videojuegos }\end{array}$ & 1.000 & .635 \\
\hline $\begin{array}{l}\text { R7 Se preocupan por } \\
\text { conocer a mis amigos }\end{array}$ & 1.000 & .510 \\
\hline $\begin{array}{l}\text { R8 Se preocupan } \\
\text { por mis problemas } \\
\text { personales }\end{array}$ & 1.000 & .663 \\
\hline $\begin{array}{l}\text { R9 Me dan confianza } \\
\text { para contarles mis } \\
\text { problemas }\end{array}$ & 1.000 & .564 \\
\hline $\begin{array}{l}\text { R10 Se preocupan por } \\
\text { mis emociones }\end{array}$ & 1.000 & .542 \\
\hline $\begin{array}{l}\text { R11 Me brindan } \\
\text { muestras de cariño }\end{array}$ & 1.000 & .621 \\
\hline $\begin{array}{l}\text { R12 En casa me siento } \\
\text { querido y protegido }\end{array}$ & 1.000 & .670 \\
\hline
\end{tabular}




\begin{tabular}{|l|l|l|}
\hline $\begin{array}{l}\text { R13 Me siento bien } \\
\text { cuando me llaman la } \\
\text { atención de manera } \\
\text { adecuada }\end{array}$ & 1.000 & .425 \\
\hline $\begin{array}{l}\text { R14 Se preocupan } \\
\text { porque no pase mucho } \\
\text { tiempo fuera de casa }\end{array}$ & 1.000 & .659 \\
\hline $\begin{array}{l}\text { R15 Procuran que } \\
\text { pasemos tiempo en } \\
\text { familia }\end{array}$ & 1.000 & .559 \\
\hline
\end{tabular}

\section{VARIANZA TOTAL EXPLICADA}

En la tabla número 4 , se puede apreciar el porcentaje de varianza por factor y el total acumulado, el valor de este último asciende prácticamente al $60 \%$ y se considera como "bueno" para la aceptación del modelo

Tabla 4. Resultado de la varianza explicada

\begin{tabular}{|c|c|c|c|c|}
\hline \multirow{5}{*}{} & $\begin{array}{c}\text { Sumas de } \\
\text { extracción de } \\
\text { Cargas al cuadrado }\end{array}$ & \multicolumn{3}{c|}{ Sumas de rotación de cargas al } \\
\cline { 2 - 5 } cuadrado
\end{tabular}




\section{GRÁFICO DE SEDIMENTACIÓN.}

En la figura número 1, se encuentra el gráfico de sedimentación como resultado de la prueba del modelo, en este se observa que en el punto de corte se encuentran los cinco factores que explican la varianza del modelo.

\section{VARIABLES LATENTES DEL MODELO}

En la tabla número 5, se observa la matriz de componentes rotados, en ella se pueden apreciar las cargas factoriales del modelo,

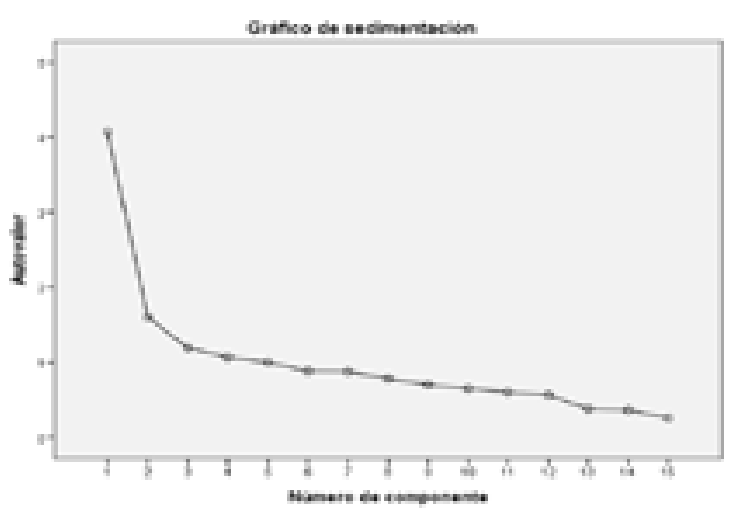

Figura 1. Gráfico de sedimentación

cada factor está compuesto por los ítems que corresponden al mismo campo semántico y que evalúan aspectos del constructo "cuidado infantil".

Tabla 5. Factores finales del modelo

\begin{tabular}{|c|c|c|c|c|c|c|}
\hline \multicolumn{6}{|c|}{ Matriz de componente rotado ${ }^{a}$} & \\
\hline & \multicolumn{5}{|c|}{ Componente } & \\
\hline & 1 & 2 & 3 & 4 & 5 & \\
\hline $\begin{array}{l}\text { R8 Se preocupan por mis problemas } \\
\text { personales }\end{array}$ & .773 & & & & & \\
\hline $\begin{array}{l}\text { R7 Se preocupan por conocer a mis } \\
\text { amigos }\end{array}$ & .635 & & & & & \\
\hline R10 Se preocupan por mis emociones & .611 & .301 & & & & \\
\hline $\begin{array}{l}\text { R9 Me dan confianza para contarles } \\
\text { mis problemas }\end{array}$ & .601 & & & & .340 & \\
\hline $\begin{array}{l}\text { R12 En casa me siento querido y } \\
\text { protegido }\end{array}$ & .309 & .746 & & & & \\
\hline R11 Me brindan muestras de cariño & & .735 & & & & \\
\hline $\begin{array}{l}\text { R13 Me siento bien cuando me llaman } \\
\text { la atención de manera adecuada }\end{array}$ & & .593 & & & & \\
\hline $\begin{array}{l}\text { R15 Procuran que pasemos tiempo en } \\
\text { familia }\end{array}$ & & .500 & & .423 & & \\
\hline R3 Se preocupan por mi higiene & & & .768 & & & \\
\hline $\begin{array}{l}\text { R4 Se preocupan por mi aspecto } \\
\text { personal }\end{array}$ & .319 & & .767 & & & \\
\hline $\begin{array}{l}\text { R5 Se preocupan porque coma } \\
\text { sanamente }\end{array}$ & & & .723 & & & \\
\hline $\begin{array}{l}\text { R14 Se preocupan porque no pase } \\
\text { mucho tiempo fuera de casa }\end{array}$ & & & & .767 & & \\
\hline
\end{tabular}




\begin{tabular}{|l|l|l|l|l|l|l|}
\hline $\begin{array}{l}\text { R6 Se preocupan por el tiempo que } \\
\text { paso en TV y videojuegos }\end{array}$ & & & & .764 & & \\
\hline $\begin{array}{l}\text { R1 Aportan dinero para mis festividades } \\
\text { escolares }\end{array}$ & & & & & .783 & \\
\hline $\begin{array}{l}\text { R2 Me apoyan con recursos para } \\
\text { realizar mis tareas }\end{array}$ & & & & & .601 & \\
\hline $\begin{array}{l}\text { Método de extracción: análisis de componentes principales. } \\
\text { Método de rotación: Varimax con normalización Kaiser. }\end{array}$ & \\
\hline La rotación ha convergido en 6 iteraciones.
\end{tabular}

La denominación de las variables latentes que resultaron de la prueba del AFE se describe a continuación:
a) Integración familiar con los ítems $\mathrm{R}^{8}$, $R^{7}, R^{10}$ y $R^{9}$.
b) Convivencia familiar con los ítems $\mathrm{R}^{12}$, $R^{11}, R^{13}$ y $R^{15}$.
c) Atención a la salud con los ítems $R^{3}, R^{4}$ y $R^{5}$.
d) Tiempo de esparcimiento con los ítems $\mathrm{R}^{14}$ y $\mathrm{R}^{6}$.
e) Apoyo económico con los ítems $R^{1}$ y $R^{2}$.
El resultado global del nivel de "cuidado infantil" que se derivó de la aplicación del Test, se puede apreciar en la tabla número 6 , en la información registrada se puede observar que el $67 \%$ de los niños equivalente a 103 participantes obtuvieron un nivel de cuidado del $100 \%$, el $25 \%$ equivalente a 39 participantes obtuvieron un nivel de cuidado del $80 \%$, el $6 \%$ equivalente a 9 participantes obtuvieron un nivel de cuidado del $60 \%$ y, solo el $2 \%$ equivalente a 3 participantes obtuvieron un nivel de cuidado del $40 \%$.

\section{RESULTADO DE LA APLICACIÓN DEL TEST.}

Tabla 5. Nivel global de cuidado infantil

\begin{tabular}{|l|l|c|c|c|c|}
\hline \multicolumn{2}{|c|}{} & Frecuencia & Porcentaje & $\begin{array}{c}\text { Porcentaje } \\
\text { válido }\end{array}$ & $\begin{array}{c}\text { Porcentaje } \\
\text { acumulado }\end{array}$ \\
\hline \multirow{4}{*}{ Válido } & $\begin{array}{l}\text { Apoyo al } \\
100 \%\end{array}$ & 103 & 48.1 & 66.9 & 66.9 \\
\cline { 2 - 6 } & Apoyo al 80\% & 39 & 18.2 & 25.3 & 92.2 \\
\cline { 2 - 6 } & Apoyo al 60\% & 9 & 4.2 & 5.8 & 98.1 \\
\cline { 2 - 6 } & Apoyo al 40\% & 3 & 1.4 & 1.9 & 100.0 \\
\cline { 2 - 6 } & Total & 154 & 72.0 & 100.0 & \\
\hline & & & & & \\
\hline
\end{tabular}




\section{CONCLUSIONES}

El cuidado infantil puede marcar una diferencia importante en el desarrollo del niño y en su actitud hacia la escuela, en este aspecto, la familia es el núcleo donde los niños y niñas aprenden a saber quienes son, a desarrollar su personalidad y donde el apoyo emocional recibido genera el ambiente en que crecen y que a la postre define elementos fundamentales para el resto de su vida.

Esta serie de conceptos han sido objeto de estudio por parte de la Psicología Positiva y fueron parte fundamental para desarrollar el Test para determinar el nivel de cuidado infantil, producto final de esta investigación.

En el proceso de construcción del Test se consideraron los argumentos anteriormente citados, y por tanto, la consideración de las variables apoyo económico, cuidado de la salud, bienestar emocional y la sana convivencia; mientras que en el proceso de validación de constructo que se desarrolló a través de la prueba de análisis factorial exploratorio se obtuvieron como producto las variables latentes Integración familiar con 4 ítems, Convivencia familiar con 4 ítems, Atención a la salud con tres ítems, Tiempo de esparcimiento con dos ítems y Apoyo económico con dos ítems.

El proceso de transición de la construcción a la validación del Test, tuvo como sustento los siguientes resultados:

a) La medida de propiedad psicométrica de fiabilidad, que arrojó un valor del estadístico del alfa de Cronbach de 0.79 , valor considerado como bastante bueno.

b) La habilitación de la prueba del AFE, de acuerdo al estadístico de KMO del cual se obtuvo un valor bastante aceptable del orden de $0.77 \mathrm{y}$, del resultado de la prueba de esfericidad de Bartlett a través de la cual se rechazó el planteamiento de la hipótesis nula. c) La adecuada definición de las variables latentes como producto de la matriz de componentes rotados que también otorgaron un toque de originalidad del Test, además de afirmar que en la matriz de componentes rotados se observó que la cantidad de componentes se ajustó a los propuestos inicialmente por la estructura del cuestionario original.

En relación al resultado global de la aplicación del Test, se encontró que el $92 \%$ de los participantes manifestaron un apoyo por parte de su familia entre el rango del $80 \%$ al $100 \%$, una de las explicaciones que de momento se encuentra para la obtención de este resultado, es que la muestra seleccionada corresponde a un nivel socioeconómico medio $\mathrm{y}$, en este sentido, es importante recordar que el contexto para el diseño y aplicación de un Test o Cuestionario es fundamental en la variación de los resultados obtenidos; obedeciendo a estas situaciones, queda pendiente la aplicación de este Cuestionario en otros contextos, además de realizar la prueba de análisis factorial confirmatorio y generar nuevas conclusiones.

\section{BIBLIOGRAFÍA}

Batthyány, K., et al. (2014). Las representaciones sociales del cuidado infantil desde una perspectiva de género. Principales resultados de la Encuesta Nacional sobre Representaciones Sociales del Cuidado. Revista Papers, Vol. 99, no. 3, pp. 335-354. doi:http://dx.doi. org/10.5565/rev/papers.686.

Caridad-Araujo, M. \& López-Boo, F. (2015). Los servicios del cuidado infantil en América Latina y el Caribe. Revista el trimestre económico, Vol. LXXXII, No. 326, pp. 249-275 
Calderón, J., et al. (2018). Análisis factorial exploratorio como método multivariante para validación de datos académicos en plataformas virtuales. Revista Lasallista de Investigación, Vol. 15, No. 2, pp. 1019. DOI: $10.22507 /$ rli.v15n2a1

Cascaes, F., et al. (2015). Estimadores de consistencia interna en las investigaciones en salud: el uso del coeficiente alfa. Rev Peru Med Exp Salud Pública, Vol. 32, N0. 1, pp. 129138

Damiani-Rodriguez, P. G. \& Vega-Dienstmaier, J. M. (2019). Selección de ítems para el desarrollo de un instrumento de evaluación de severidad de trastornos mentales en pacientes sin psicosis o déficit cognitivo. Revista de NeuroPsiquiatría, Vol. 82, núm. 1, pp. 1-17

Lloret, S., et al. (2014). El análisis factorial exploratorio de los ítems: una guía práctica, revisada y actualizada. Revista Anales de psicología, Vol. 30, No. 3, pp. 1151-1169. https://doi.org/10.6018/ analesps.30.3.199361

Maese, J de D., et al. (2016). Coeficiente alfa de Cronbach para medir la fiabilidad de un cuestionario difuso. Revista Culcyt. Instrumentos de Medición, Año 13, No. 59, pp. 146-156

Moliner, L., et al. (2017). Diseño, validación $y$ análisis factorial exploratorio $y$ confirmatorio de la escala de actitud Cohesiona para la evaluación de la eficacia de los talleres de habilidades cooperativas. Revista Estudios Pedagógicos, Vol. XLIII, No. 1, pp. 213234
Montero, I. \& León, O. (2007). A guide for naming research studies in Psychology. (E. Universidad Autonóma de Madrid, Ed.) International Journal of Clinical and Health Psycology, 7 (3), pp. 847- 862.

Montoya, O. (2007). Aplicación del análisis factorial a la investigación de mercados. Caso de estudio. Revista Scientia et Technica, Año 13, No. 35, pp. 281, 286

Pérez, E. R. \& Medrano, L. (2010). Análisis Factorial Exploratorio: Bases Conceptuales y Metodológicas. Revista Argentina de Ciencias del Comportamiento (RACC), Vol. 2, No. 1, pp. $58-66$

Razeto, A. (2016). El involucramiento de las familias en la educación de los niños. Cuatro reflexiones para fortalecer la relación entre familias y escuelas. Revista Páginas de Educación, Vol. 9, No. 2, pp. 1-26

Royo, P., et al. (2012). Fundamentals for the positive development of the child. Pulso: Revista en Educación, Vol. 35, pp. 1329. 


\section{APÉNDICES}

\section{Apéndice 1}

Instrumento inicial validado por expertos

\begin{tabular}{|c|c|c|c|c|c|}
\hline \multirow{2}{*}{$\begin{array}{l}\text { No. } \\
\text { Ítem }\end{array}$} & \multirow[t]{2}{*}{ Descripción } & \multicolumn{4}{|c|}{ Nivel } \\
\hline & & $\begin{array}{c}1 \\
100 \%\end{array}$ & $\begin{array}{c}2 \\
80 \%\end{array}$ & $\begin{array}{c}3 \\
60 \%\end{array}$ & $\begin{array}{c}4 \\
40 \%\end{array}$ \\
\hline $\mathrm{R} 1$ & Aporta dinero para mis festividades escolares & & & & \\
\hline $\mathrm{R} 2$ & $\begin{array}{l}\text { Cuento con un espacio adecuado para } \\
\text { realizar mis tareas }\end{array}$ & & & & \\
\hline R3 & Cuento con recursos para realizar mis tareas & & & & \\
\hline $\mathrm{R} 4$ & Me apoya para hacer mis tareas & & & & \\
\hline R5 & Se preocupa por lo que como fuera de casa & & & & \\
\hline $\mathrm{R} 6$ & Se preocupa por mi higiene particular & & & & \\
\hline $\mathrm{R} 7$ & Se preocupa por mi salud & & & & \\
\hline $\mathrm{R} 8$ & Se preocupa porque tenga ropa limpia & & & & \\
\hline R9 & Se preocupa porque coma sanamente & & & & \\
\hline R10 & $\begin{array}{l}\text { Se preocupa por llevarme lonche a la hora } \\
\text { del recreo }\end{array}$ & & & & \\
\hline R11 & $\begin{array}{l}\text { Se preocupa por el tiempo que paso en TV y } \\
\text { videojuegos }\end{array}$ & & & & \\
\hline $\mathrm{R} 12$ & Se preocupa por conocer a mis amigos & & & & \\
\hline $\mathrm{R} 13$ & Se preocupa por mis problemas personales & & & & \\
\hline R14 & Me da confianza para contarle mis problemas & & & & \\
\hline R15 & Se preocupa por mis emociones & & & & \\
\hline R16 & Me brindan muestras de cariño & & & & \\
\hline $\mathrm{R} 17$ & En casa me siento querido y protegido & & & & \\
\hline R18 & $\begin{array}{l}\text { Me siento bien cuando me llaman la atención } \\
\text { de manera adecuada }\end{array}$ & & & & \\
\hline R19 & Disfrutamos tiempo libre en familia & & & & \\
\hline R20 & $\begin{array}{l}\text { Se preocupa porque no pase mucho tiempo } \\
\text { fuera de casa }\end{array}$ & & & & \\
\hline R21 & Procura que pasemos tiempo en familia & & & & \\
\hline
\end{tabular}




\section{Apéndice 2}

Instrumento final validado por expertos

\begin{tabular}{|c|c|c|c|c|c|}
\hline \multirow{2}{*}{$\begin{array}{c}\text { No. Ítem } \\
\text { (reordenado) }\end{array}$} & \multirow[t]{2}{*}{ Descripción } & \multicolumn{4}{|c|}{ Nivel } \\
\hline & & $\begin{array}{c}1 \\
100 \%\end{array}$ & $\begin{array}{c}2 \\
80 \%\end{array}$ & $\begin{array}{c}3 \\
60 \%\end{array}$ & $\begin{array}{c}4 \\
40 \%\end{array}$ \\
\hline R1 & Aporta dinero para mis festividades escolares & & & & \\
\hline $\mathrm{R} 2$ & Cuento con recursos para realizar mis tareas & & & & \\
\hline R3 & Se preocupa por mi salud & & & & \\
\hline R4 & Se preocupa porque tenga ropa limpia & & & & \\
\hline R5 & Se preocupa porque coma sanamente & & & & \\
\hline R6 & $\begin{array}{l}\text { Se preocupa por el tiempo que paso en TV y } \\
\text { videojuegos }\end{array}$ & & & & \\
\hline R7 & Se preocupa por conocer a mis amigos & & & & \\
\hline R8 & Se preocupa por mis problemas personales & & & & \\
\hline $\mathrm{R} 9$ & Me da confianza para contarle mis problemas & & & & \\
\hline R10 & Se preocupa por mis emociones & & & & \\
\hline R11 & Me brindan muestras de cariño & & & & \\
\hline R12 & En casa me siento querido y protegido & & & & \\
\hline R13 & $\begin{array}{l}\text { Me siento bien cuando me llaman la atención } \\
\text { de manera adecuada }\end{array}$ & & & & \\
\hline R14 & $\begin{array}{l}\text { Se preocupa porque no pase mucho tiempo } \\
\text { fuera de casa }\end{array}$ & & & & \\
\hline R15 & Procura que pasemos tiempo en familia & & & & \\
\hline
\end{tabular}

